\title{
ATLAS Trigger Algorithms for General Purpose Graphics Processor Units
}

\author{
Ademar Tavares Delgado and Dmitry Emeliyanov on behalf of ATLAS Collaboration
}

\begin{abstract}
We present ATLAS Trigger algorithms developed to exploit General Purpose Graphics Processor Units (GPGPU). ATLAS is a particle physics experiment located on the LHC collider at CERN. The ATLAS Trigger system has two levels, hardware-based Level 1 and the High Level Trigger implemented in software running on a farm of commodity CPU. Performing the trigger event selection within the available farm resources presents a significant challenge that will increase following future LHC upgrades. GPGPUs are being evaluated as a potential solution for trigger algorithms acceleration. Key factors determining the potential benefit of this new technology are the relative execution speed-up, the number of GPUs required and the relative financial cost of the selected GPU.

We have developed a trigger demonstrator which includes algorithms for reconstructing tracks in the Inner Detector and Muon Spectrometer and clusters of energy deposited in the Calorimeter. A "client-server" architecture is used to integrate the GPU-accelerated code running on the server side with multiple clients running the remaining serial code on the CPUs. We give a description of the algorithms implemented and present measurements that compare the performance of various algorithm configurations.
\end{abstract}

\section{INTRODUCTION}

A TLAS [1] is one of the two general purpose detectors located at the Large Hadron Collider (LHC) [2] at CERN. ATLAS has a cylindrical layout which includes several subdetector systems with a total of about $10^{8}$ readout channels. The Inner Detector (ID) tracker is located inside a solenoid magnet and contains three different detector technologies. Pixel detectors form the innermost layers surrounded by the Semiconductor Tracker (SCT) and Transition Radiation Tracker (TRT) respectively. The Calorimeter systems surrounding the ID consist of the Liquid Argon (LAr) Electromagnetic calorimeter and the Hadronic calorimeter. Toroidal magnets and muon detectors form the Muon Spectrometer at the outermost layers of the ATLAS detector.

The ATLAS Trigger and Data Acquisition system (TDAQ) is shown schematically in Fig. 1 with the event and data rates for the current Run 2 and future Run 3. The trigger system which is responsible for selecting events of interest has two levels - the custom hardware-based Level 1 and the HighLevel Trigger (HLT) which runs software algorithms for event reconstruction and selection on a farm of commodity CPUs.

During Run 3 which starts in 2021 the LHC will be operated at a higher centre-of-mass energy and instantaneous luminosity than in 2016. It is expected that the average number of

Ademar Tavares Delgado is with Laboratorio de Instrumentacao e Fisica Experimental de Particulas, LIP, Lisboa, Portugal

Dmitry Emeliyanov is with STFC Rutherford Appleton Laboratory, Harwell Science and Innovation Campus, Didcot, UK

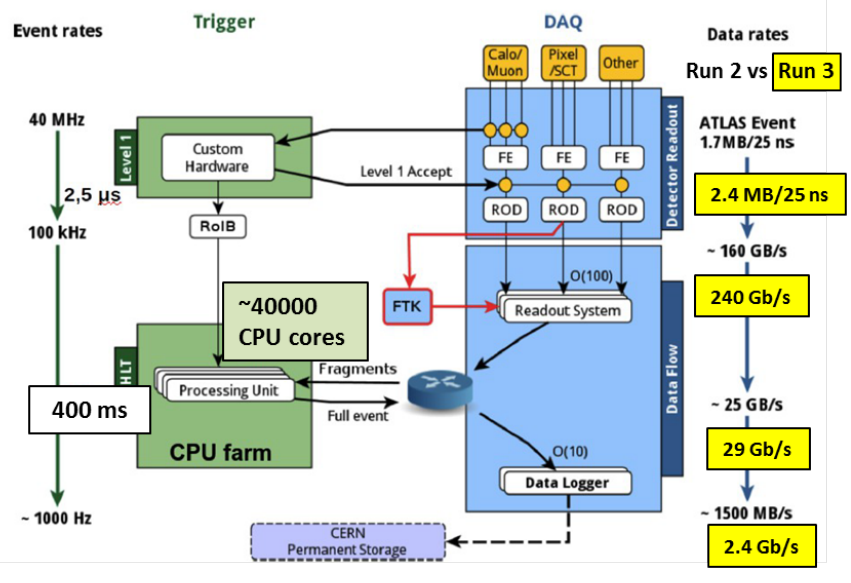

Fig. 1: General structure of the ATLAS TDAQ, event processing and data rates for the LHC Run 2 and Run 3.

simultaneous interactions (event pile-up) will approximately double compared to 2016. Due to the larger event pile-up and higher detector occupancy the High-Level Trigger will have to handle more complex events within the same limited time budget. The main problem is that, because of the combinatorial nature of event reconstruction algorithms, the HLT processing time increases exponentially with the level of event pile-up. At the same time, the number of CPUs in the HLT farm is limited by constraints of physical space, electrical power and cooling capacity. Using General-Purpose GPUs as accelerators for the HLT farm is considered as one of the possible options in order to provide more processing power for ATLAS Trigger within the above constraints. To evaluate the feasibility of this approach we have developed a Trigger GPU demonstrator which includes various event reconstruction algorithms implemented on NVIDIA Kepler GPUs. The algorithms selected for the demonstrator have inherent data parallelism and thus are suitable for massively parallel architectures such as GPUs. The ultimate goal of this work is to provide an estimate of the achievable event processing rate per unit cost including both, the cost of hardware and effort.

The remainder of the paper is organized as follows. The software architecture which integrates GPU-accelerated algorithms with the ATLAS HLT event processing framework is presented in Section II. The trigger algorithms implemented on GPU are described in Section III. The performance of the GPU-accelerated algorithms is presented and discussed in Section IV. 


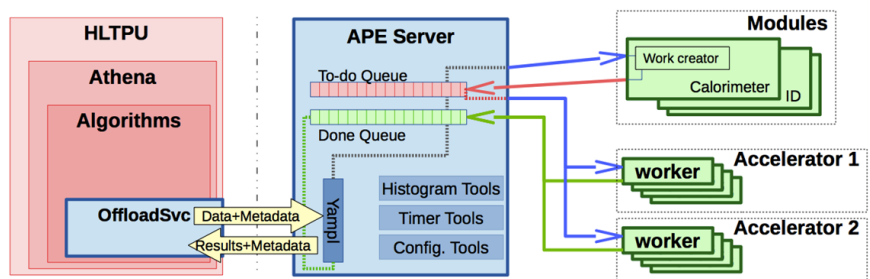

Fig. 2: The "client-server" architecture for GPU-accelerated event processing in the ATLAS HLT.

\section{GPU-ACCELERATED EVENT PROCESSING}

In the ATLAS HLT, one HLT process (HLTPU) is executed on each virtual CPU core in the HLT farm. Each HLTPU processes separate events independently. The HLTPU runs the ATLAS event reconstruction framework referred to as Athena and interfaces it with the DAQ system.

In order to incorporate GPGPUs into the HLT event processing workflow we employed the "client-server" architecture shown in Fig. 2.

In the context of this architecture, a client is a HLTPU process which runs a sequence of HLT algorithms. The "clientserver" approach abstracts the accelerator hardware from the clients and allows the sharing of diverse GPU resources among many active HLTPU processes.

The server is a separate process called the Accelerator Process Extension (APE) which manages requests from multiple clients and executes the accelerated algorithms on a GPU. The server has a modular structure with plug-in modules which implement the algorithms and can support various GPU hardware/API technologies, for instance OpenCL or NVIDIA CUDA.

The client interacts with the server using a service (OffloadSvc in Fig. 2) which handles InterProcess Communication (IPC) and conversion of data formats between the original, Athena-specific, data classes and customized, GPU-friendly, C-like data structures.

\section{GPU-BASED TRIGGER ALGORITHMS}

Analysis of the HLT processing time identified several time consuming algorithms suitable for parallelisation. Two of them, topological Calorimeter clustering and Inner Detector track seeding, are described below.

\section{A. GPU-accelerated Calorimeter cell clustering}

The significant execution time of the Topological Clustering (TopoCluster) [3], largest among the calorimeter algorithms, and its inherent parallelism makes it a good candidate for GPU acceleration.

The algorithm joins the calorimeter detection units, known as cells, to form three-dimensional energy depositions (clusters) whilst suppressing the noise contributions. The noise suppression is achieved by making the clustering dependent on cell and their neighbours energy significance in terms of the Signal-over-Noise $(\mathrm{S} / \mathrm{N})$ ratio of the energy deposition and the average electronics and pile-up noise in cells.
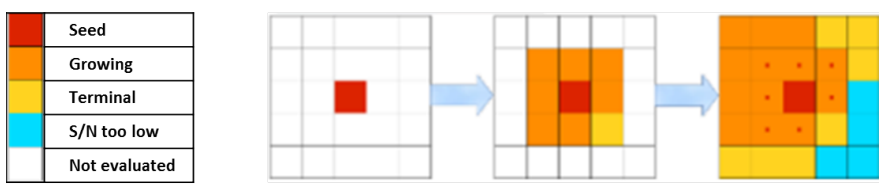

Fig. 3: The topological clustering of the Calorimeter cells.

The TopoCluster algorithm applies three different programmable $\mathrm{S} / \mathrm{N}$ thresholds in order to classify the cells in three groups: Seed cells which have $\mathrm{S} / \mathrm{N}$ above the highest $\mathrm{S} / \mathrm{N}$ threshold, Growing cells which pass the intermediate threshold, and Terminal cells which pass the lowest threshold. By default, the thresholds have the values 4,2 , and 0 , respectively.

Once cells are classified the algorithm proceeds with the cluster growing phase. Each Seed-cell initiates a new cluster, as shown in Fig. 3. The algorithm processes the cluster candidates one by one by iteratively including neighbours of Seed or Growing cells. Two different clusters are merged if they share a Seed- or Growing-cell.

The GPGPU version of this algorithm, the Topological Automaton Clustering (TAC), is based on a cellular automaton (CA) approach. In order to maintain properties of the original algorithm the CA evolution rules reflect the cluster growing rules of the TopoCluster algorithm.

The TAC algorithm consists of three kernels: the cell classification and cell pair building kernel, the unique-tag generator kernel, and the cluster-growing kernel.

The TAC starts with a kernel that does parallel S/N-based cell classification in which each GPU thread evaluates one calorimeter cell. The CA workspace consists of pairs of neighbouring cells formed only by Seed and Growing cells, as these are only the ones that can make clusters grow. This provides efficient data reduction, by removing from further computation inactive pairs. The cell pairing also ensures an evenly distribution of workload across all GPU threads and creates enough parallel work to keep all GPU threads busy.

The second kernel sorts the Seed cells by $\mathrm{S} / \mathrm{N}$, in increasing order, and assigns to each of them a unique cluster tag equal to the Seed-cell position in the ordered list. This unique tag is an essential component of the cellular automaton algorithm.

The third kernel performs the cluster growing. Each GPU thread evaluates a pair of cells and propagates the largest cluster tag in accordance with the following rules:

- A Seed- or a Growing-cell propagates its tag to neighbouring cells with smaller tags.

- Clusters can only have cells above the Terminal threshold.

- Clusters are merged if they share a Seed-cell or a Growing-cell.

If a new tag is proposed to a Seed- or Growing-cell that have been already assigned to another cluster then a tag pair is formed for the subsequent merging step. The algorithm continues evaluating pairs of cells for as long as there are cell tag changes. At the end of each loop the merging step is executed, processing all cells, each one in a different thread. If the cluster they belong to is marked to be merged, their tag is replaced with the larger one of the merging tag pair. 


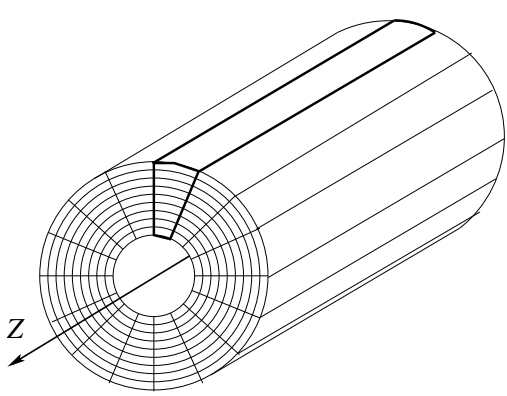

(a) $\phi$-sectors

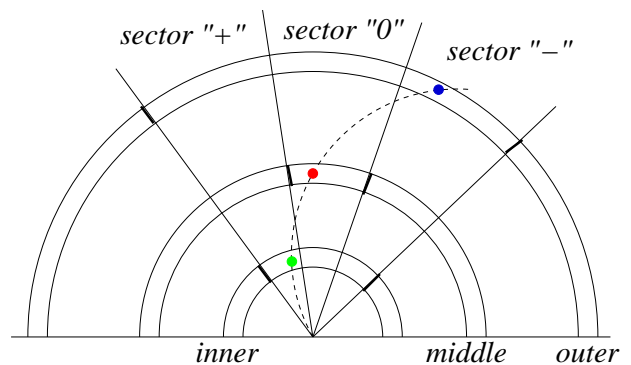

(b) triplet in $r-\phi$-projection

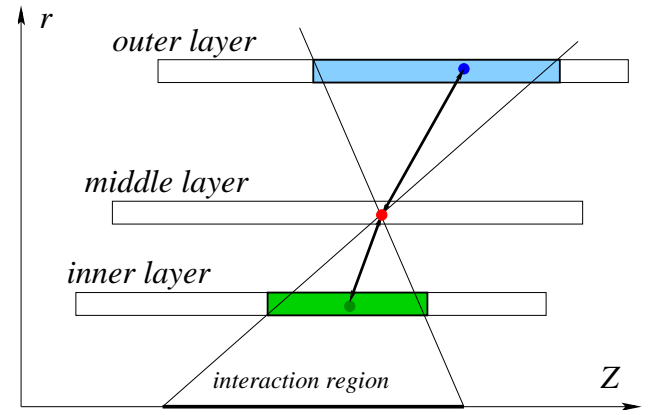

(c) doublets and triplet in $r-Z$-projection

Fig. 4: Formation of spacepoint doublets and triplets.

The list of cells with their cluster tag and the clusters properties are the results sent to the HLT client. The client then converts the response to the Athena-specific data structures. The next TopoCluster step, the cluster splitting, is then executed using a CPU-based algorithm.

\section{B. GPU-accelerated Inner Detector tracking}

The Inner Detector (ID) track reconstruction algorithm selected for GPU-based acceleration consists of the two parts, track-seeding and seeded track-following [4]. In the Trigger GPU demonstrator only the first part was implemented on a GPU while the second part was executed on a CPU.

The track-seeding algorithm creates triplets of spacepoints (SP) referred to as track-seeds. As illustrated in Fig. 4a the spacepoints are arranged into wedge-shaped sectors determined by the spacepoint $\phi$-coordinate. The sector width is chosen so that all spacepoints on a track with curvature corresponding to the transverse momentum $p_{T}$ above $1 \mathrm{GeV}$ end up in at most 3 neighbouring sectors (Fig. 4b). For each spacepoint in a $\phi$-sector except those in the innermost Pixel layer the algorithm assumes it to be the "middle" spacepoint of a triplet and then searches for "inner" (at lower radii) and "outer" (at higher radii) spacepoints in the same sector and the two neighbouring sectors. The search is restricted by imposing a cut on the $z$-position of the "inner" and "outer" spacepoints. These are selected from $z$-intervals obtained by "projecting" the luminous region through the "middle" spacepoint as illustrated in Fig. 4c.

The GPU-based implementation of the track seeding algorithm includes three NVIDIA CUDA kernels.

First, the number of inner and outer doublets are calculated for each middle spacepoint and then the doublet storage in the GPU device memory is allocated in accordance with these numbers. The second kernel creates the doublets and stores them in the "collections" - sub-intervals inside a Structure-ofArrays (SoA) in the GPU device memory. The third kernel is responsible for combining the doublets from the SoA into triplets and further data reduction by applying various kinematical and quality cuts.

\section{RESULTS AND DISCUSSION}

The GPU-accelerated algorithms have been tested using a system consisting of a host with two Intel(R) Xeon(R) E5-

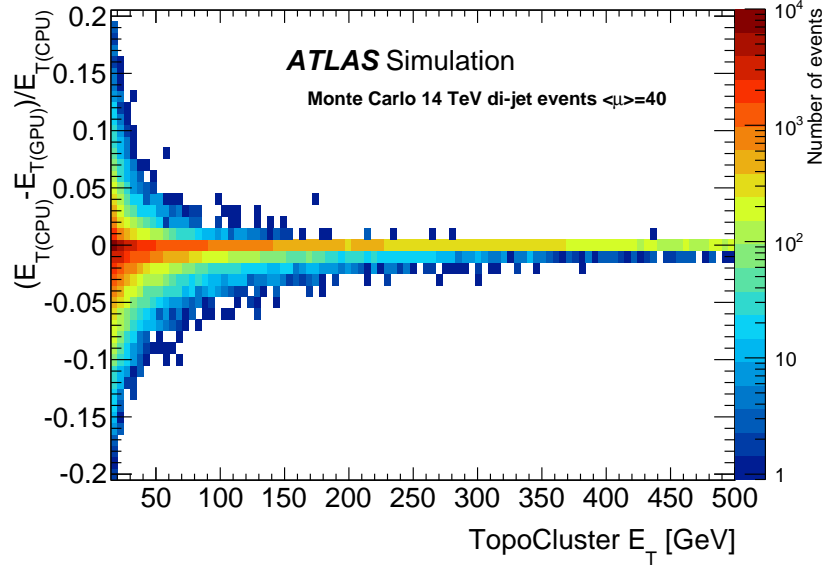

Fig. 5: The relative transverse energy difference between the clusters reconstructed by the GPU and CPU algorithms [5].

2695 v3 14-core CPUs with a clock speed of $2.3 \mathrm{GHz}$ and two NVIDIA GK210GL GPUs in a Tesla K80 module. The host machine had 28 physical CPU cores in total which allowed running up to 56 independent HLT processes using HyperThreading.

For most of the tests described below a simulated ATLAS $t \bar{t}$ decay dataset converted to a raw detector output format (bytestream) was used. This dataset has an average of $46 \mathrm{su}-$ perimposed minimum bias events per simulated collision corresponding to instantaneous luminosity of $1.7 \times 10^{34} \mathrm{~cm}^{-2} \mathrm{~s}^{-1}$.

\section{A. GPU-accelerated Calorimeter clustering}

The excellent agreement between the GPU and CPU calorimeter clustering algorithms is illustrated by Fig. 5 which shows the relative transverse energy difference between the calorimeter clusters reconstructed using the standard CPU cell clustering algorithm and the matching clusters found by the algorithm ported to GPU. Clusters are matched using the unique cluster identifiers which are generated identically for both, GPU and CPU, algorithms. The data sample used consists of QCD di-jet events with leading-jet transverse momentum above $20 \mathrm{GeV}$ and a pile-up of 40 simultaneous interactions per bunch-crossing. 

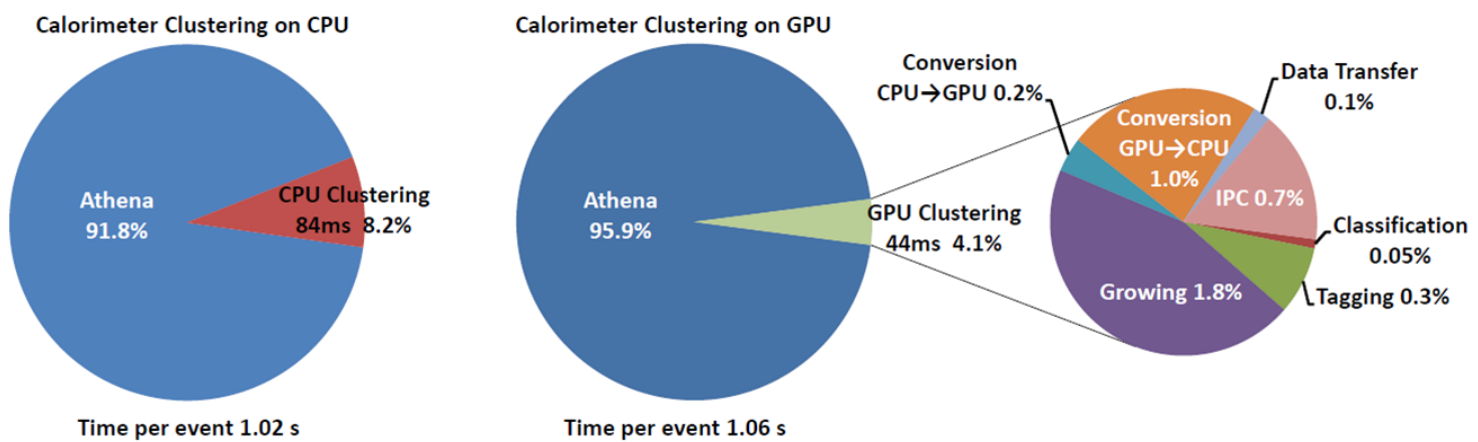

Fig. 6: Breakdown of the time per event for the HLT process running Calorimeter clustering on CPU and offloaded to a GPU.

TABLE I: Topological clustering speed-up factors.

\begin{tabular}{c|c|c|c}
\hline Dataset & di-jets & $t \bar{t}$ & $t \bar{t}$ \\
\hline Pile-up & 40 & 46 & 138 \\
\hline Speed-up & 1.3 & 2 & 2 \\
\hline
\end{tabular}

The timing of the GPU-based clustering is shown in Fig. 6 which also shows the time for the Classification, Tagging, and Clustering kernels running on the GPU (GPU execution) and the overhead associated with offloading the work to a GPU (Other). The overhead comprises the time to convert datastructures between CPU and GPU data-formats, the data transfer time between CPU and GPU and the IPC time that accounts for the transfer of data between the HLT clients and the server. Note that there is a small increase in the execution time of the non-accelerated code when the calorimeter clustering is offloaded to GPU.

As Table I shows, the speed-up factor of the Calorimeter clustering increases with data multiplicity and pile-up level.

The split timing analysis (Fig. 6) indicates a relatively large overhead caused by conversion of the clustering output back to Athena-specific data structures required for subsequent processing. The purely algorithmic speed-up factor of the TAC algorithm is about 3.8 , reducing to 2 if the data conversion overhead is taken into account.

\section{B. GPU-accelerated ID tracking}

The track reconstruction efficiency was measured with respect to the reference set of simulated tracks with $p_{T} \geq 1 \mathrm{GeV}$ and $|\eta| \leq 2.5$. The closest HLT track within a cone $\Delta R=$ $\sqrt{\Delta \eta^{2}+\Delta \phi^{2}} \leq 0.05$ surrounding a reference track was selected as a correctly reconstructed matching track.

As Fig. 7 shows, the accelerated ID tracking has the same track finding efficiency as the CPU-only algorithm. The excellent agreement between the GPU and CPU algorithms is demonstrated in Fig. 8 which shows the transverse impact parameter distributions for the simulated tracks correctly reconstructed by the GPU-accelerated tracking algorithm and the CPU-only algorithm.

The breakdown of the algorithm execution time per event is shown in Fig. 9 where the time fractions for the Counting, Doublet Making and Triplet Making kernels running on the

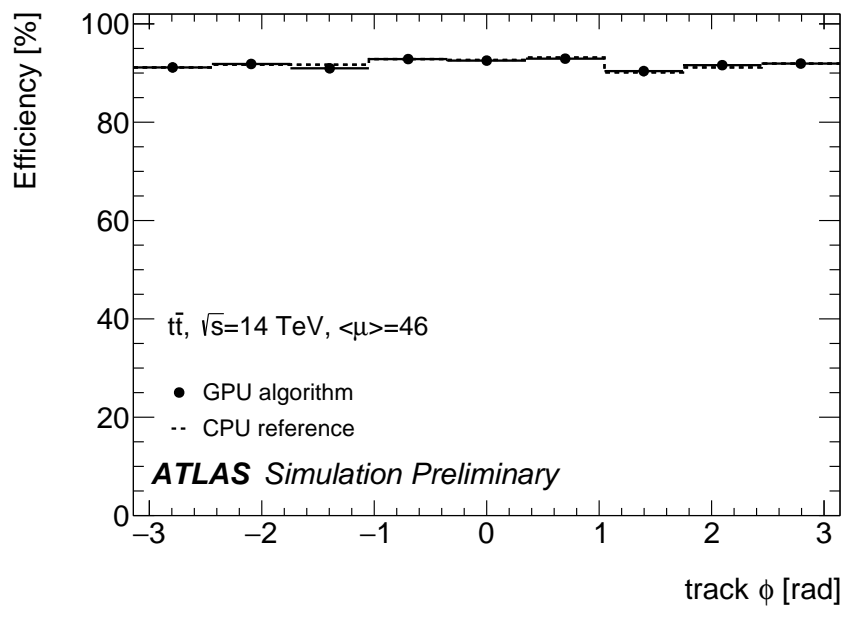

Fig. 7: Track reconstruction efficiency as a function of simulated track azimuth for the GPU-accelerated tracking algorithm and the CPU-only algorithm [5].

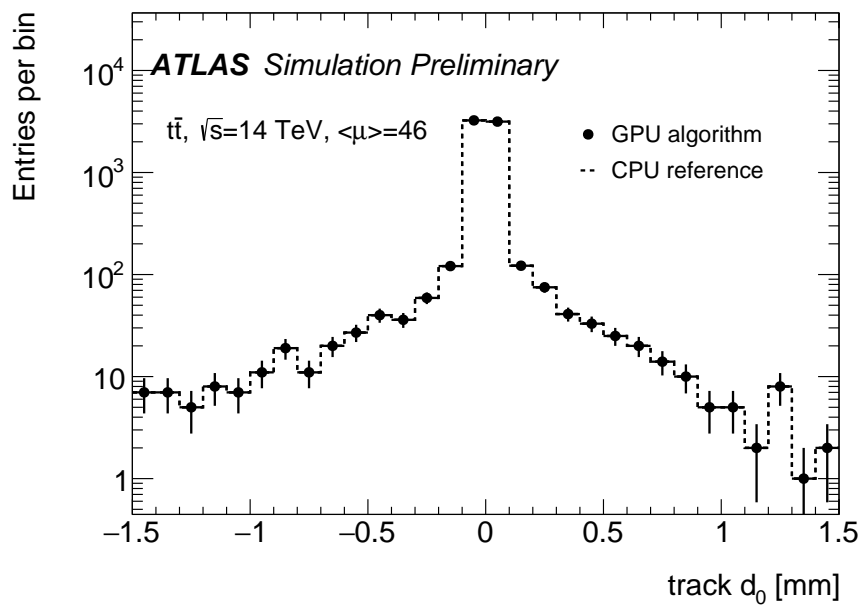

Fig. 8: The comparison of the transverse impact parameter distributions of the tracks reconstructed by the GPU and CPU algorithms [5].

GPU (GPU execution) and the overhead associated with offloading the work (Other) are shown. 

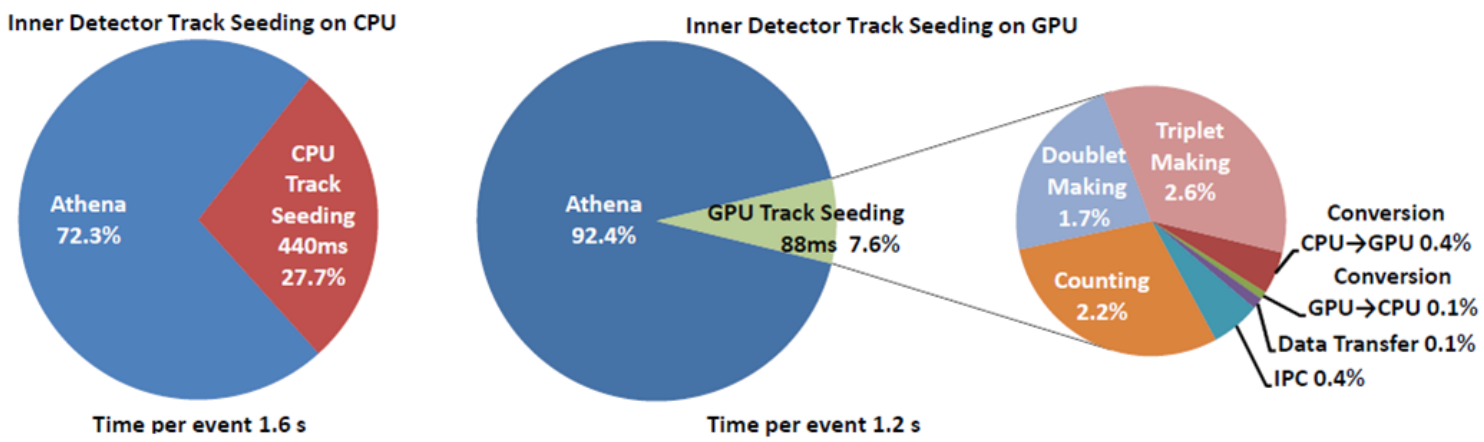

Fig. 9: Breakdown of the time per event for the HLT process running ID track seeding on the CPU or offloaded to a GPU.

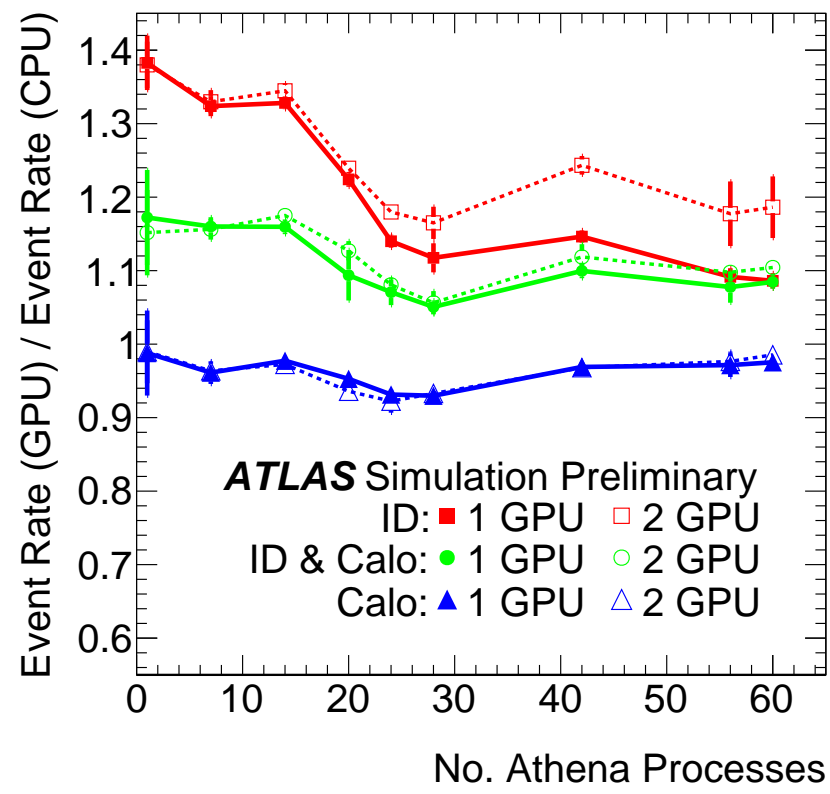

Fig. 10: The ratio of event processing rates with GPU acceleration to the CPU-only rates as a function of the number of client processes [5].

These timing measurements were made with a single GPU and 12 HLT client processes configured to run only ID tracking. As can be seen from Fig. 9, the track seeding is 5 times faster if run on GPU. The purely algorithmic speed-up factor excluding the IPC and data conversion overhead is about 5.8. This speed-up results in reduction of total processing time per event by $25 \%$.

\section{Event processing rate}

The overall gain in event processing rate is illustrated by Fig. 10. Separate tests were performed with Athena jobs configured to execute only Inner Detector Tracking (ID), only Calorimeter topological clustering (Calo) or both (ID \& Calo). The system was configured to either perform the work on the CPU or offload to one or two GPU.

The ID track seeding takes about $30 \%$ of event processing time on CPU and is accelerated by about a factor of 5 on GPU.

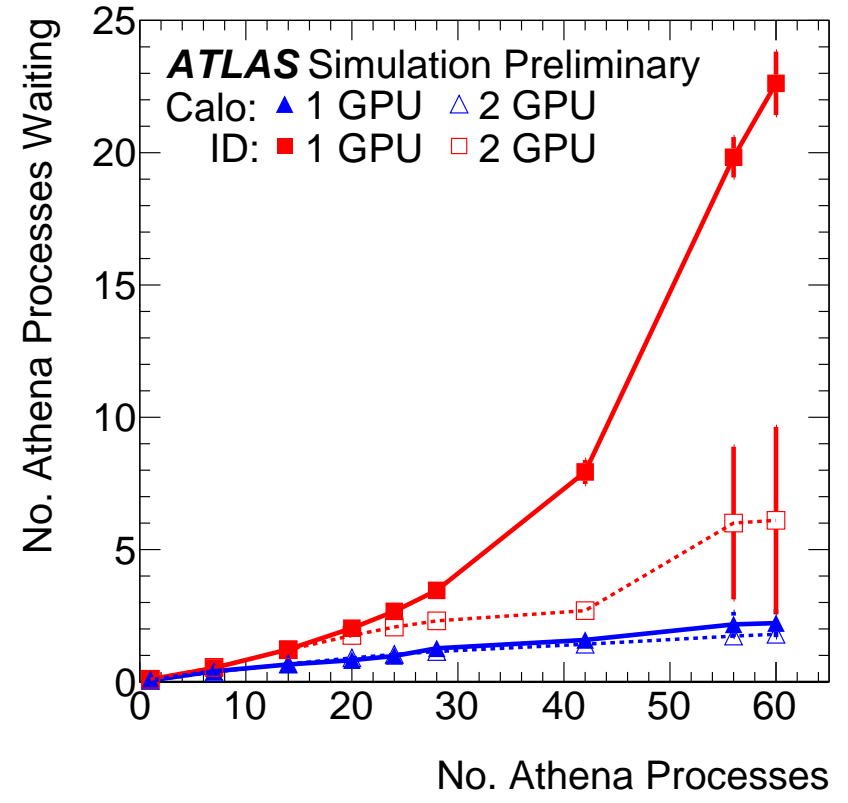

Fig. 11: The APE server waiting queue depth illustrating the occupancy of the GPU [5].

As a result throughput increases by about $35 \%$ with GPU acceleration for up to 14 client processes. The Calorimeter clustering algorithm takes about $8 \%$ of event processing time on CPU and is accelerated by about a factor 2 on GPU, however the effect of the acceleration is offset by a small increase in the time of the non-accelerated code and as a result a small decrease in speed is observed with offloading to GPU.

The time-averaged mean number of HLT processes (clients) waiting for the result of the GPU computation to be returned by the APE server as a function of the number of clients is shown in Fig. 11. The GPU-accelerated ID track seeding takes about $8 \%$ of the total event processing time and so the average number of HLT clients waiting is less than 1 for up to about 12 HLT clients. The GPU-based calorimeter clustering takes about $4 \%$ of event processing time on CPU and so the average number of HLT clients waiting is less than 1 for up to about 25 HLT clients.

As can be seen from Fig. 10, the observed gain in event 
processing rate is $20-40 \%$ depending on the number of HLT clients. The server occupancy plot (Fig. 11) indicates that for ID tracking jobs a single GPU can efficiently serve up to 14 HLT clients.

\section{CONCLUSION}

Following the LHC upgrade the ATLAS trigger algorithms will have to handle more complex events within a limited time budget. To meet the demand for computing power, ATLAS is considering the possible use of GPGPUs in the trigger. To make the existing algorithms suitable for GPUbased acceleration they have been re-implemented as modules within the Trigger GPU demonstrator software integrated with the ATLAS event processing framework.

First tests of GPU-based tracking and calorimeter reconstruction demonstrated acceleration factors about 5 for track seeding and 2 for topological cluster formation with the same physics performance.
The overall gain in event throughput was $20-40 \%$ depending on the number of clients sharing a GPU. The event processing rate can be improved if more code is ported to the GPU and data conversion overheads reduced by using efficient data structures suitable for both GPU and CPU.

Further tests will be conducted in order to evaluate cheaper gaming videocards based on the latest NVIDIA Pascal GPU architecture.

\section{REFERENCES}

[1] ATLAS Collaboration JINST 3 S08003, 2008.

[2] L. Evans and P. Bryant JINST 3 S08001, 2008.

[3] W. Lampl et al. ATLAS Note, 2008. http://cds.cern.ch/record/1099735

[4] A. Salzburger, Journal of Physics: Conference Series 664 (2015) 072042.

[5] ATLAS Experiment - public results: https://twiki.cern.ch/twiki/bin/view/ AtlasPublic/TriggerSoftwareUpgradePublicResults 